\title{
A collocation-based multi-configuration time-dependent Hartree method using mode combination and improved relaxation
}

\author{
Robert Wodraszka ${ }^{\mathrm{a}}$ and Tucker Carrington Jr. ${ }^{\mathrm{b}}$ \\ Chemistry Department, Queen's University, \\ Kingston, Ontario K ${ }^{\prime} \mathrm{L}$ 3N6, Canada
}

(Dated: March 31, 2020)

\begin{abstract}
Although very useful, the original multi-configuration time-dependent Hartree (MCTDH) method has two weaknesses: (1) its cost scales exponentially with the number of atoms in the system; (2) the standard MCTDH implementation requires that the PES be in sum-of-product (SOP) form in order to reduce the cost of computing integrals in the MCTDH basis. One way to deal with (1) is to lump coordinates into groups. This is mode combination (MC). One way to deal with (2) is to reformulate MCTDH using collocation so that there are no integrals. In this paper we combine $\mathrm{MC}$ and collocation to formulate a mode combination collocation multi-configuration time-dependent Hartree method (MC-C-MCTDH). In practice, its cost does not scale exponentially with the number of atoms and it can be used with any general potential energy surfaces (PES); the PES need not be a SOP and need not have a special form. No integrals and hence no quadratures are necessary. We demonstrate the accuracy and efficiency of the new method by computing vibrational energy eigenstates of methyl radical, methane, and acetonitrile. To do this, we use MC-C-MCTDH with a variant of improved relaxation, derived by evaluating a residual at points. Because the MC basis functions are multivariate, collocation points in multidimensional spaces are required. We use two types of collocation points: 1) DVR (discrete variable representation)-like points obtained from (approximate) simultaneous diagonalisation of matrices; and 2) Leja points, which are known to be good interpolation points, determined from a generalised recipe suitable for any basis.
\end{abstract}

\footnotetext{
${ }^{a}$ Electronic address: Robert.Wodraszka@chem.queensu.ca

b Electronic address: Tucker.Carrington@queensu.ca
} 


\section{INTRODUCTION}

Multi-configuration time-dependent Hartree (MCTDH) methods are prominent approaches for solving the Schrödinger equation to study the dynamics of nuclei. It is well established that they can be used to compute accurate rate constants, cross sections, spectra etc. ${ }^{1-14}$ The original MCTDH method ${ }^{15,16}$ has two important weaknesses. First, both the memory and the time costs of an MCTDH calculation scale exponentially with the number of nuclei. Second, calculating matrix-vector products in the MCTDH basis is, if the Hamiltonian is not a sum of products, difficult. The mode combination-collocation MCTDH method introduced in this paper mitigates both these weaknesses.

The key MCTDH idea is to use a direct product basis built from variationally optimised univariate functions, the so-called single-particle functions (SPFs), to represent a wavefunction,

$$
\Psi\left(x_{1}, \ldots, x_{D}, t\right)=\sum_{i_{1}=1}^{n_{1}} \ldots \sum_{i_{D}=1}^{n_{D}} A_{i_{1}, \ldots, i_{D}}(t) \varphi_{i_{1}}^{(1)}\left(x_{1}, t\right) \varphi_{i_{2}}^{(2)}\left(x_{2}, t\right) \ldots \varphi_{i_{D}}^{(D)}\left(x_{D}, t\right) .
$$

The SPFs are determined by expanding them in a time-independent basis

$$
\varphi_{i_{k}}^{(k)}\left(x_{k}, t\right)=\sum_{f_{k}=1}^{N_{k}} c_{f_{k}, i_{k}}^{(k)}(t) \chi_{f_{k}}^{(k)}\left(x_{k}\right) \quad \forall k=1 \ldots D .
$$

In many applications, due to the optimisation of the $\varphi_{i_{k}}^{(k)}\left(x_{k}, t\right), \prod_{k=1}^{D} n_{k}<<\prod_{k=1}^{D} N_{k}$ and the MCTDH basis is much smaller than the direct product basis made from the primitive $\chi_{f_{k}}^{(k)}\left(x_{k}\right)$ bases. When the Hamiltonian is a sum of products, it is easy to implement $\mathrm{MCTDH}^{16}$ so that the memory cost scales as $n^{D}$ and the computation time scales as $n^{D+1}$.

Mode combination (MC) is an effective way of dealing with the cost weakness. ${ }^{17} \mathrm{MC}$ makes it possible to deal with very large problems. ${ }^{3,18}$ It reduces both the memory cost and the computation time. ${ }^{1,19}$ MC uses multivariate SPFs. The multivariate SPFs are obtained by lumping several coordinates into a "logical" coordinate. The memory cost of a MC calculation (unless solving for the SPFs dominates) scales as $\tilde{n}^{p}$ and the computation time scales as $\tilde{n}^{p+1}$, where $\tilde{n}$ is the required number of SPFs and $p$ is the number of logical coordinates, known as particles. If the coupling between coordinates within logical coordinates is stronger than the coupling between coordinates in different logical coordinates, then MC significantly reduces the cost of an MCTDH calculation. So-called "contracted" basis 
functions, often used to compute spectra, ${ }^{20-23}$ and mode-combined SPFs are similar in that both incorporate coupling into the basis functions. In practice, it is often most efficient to combine two to four coordinates because if more are combined then the cost of computing the SPFs becomes too high. In this paper, we use the MC idea.

Although they are not the subject of this paper, there are two other established techniques for reducing the memory cost and the computation time. The first is multi-layer (ML-)MCTDH, ${ }^{24-26}$. Like MC, ML uses multivariate SPFs, however, they are determined recursively. SPFs are arranged in a tree and SPFs higher in the tree are represented in bases made from SPFs that are lower in the tree. The second is pruned (P-)MCTDH. ${ }^{27-30} \mathrm{In}$ $\mathrm{P}-\mathrm{MCTDH}$, functions that are not required are removed from the MCTDH direct product basis. We have shown that in some cases P-MCTDH is as efficient as ML-MCTDH. ${ }^{28}$

Irrespective of whether one uses standard MCTDH, MC-, ML-, or P-MCTDH, one must confront the second weakness. It is important to efficiently evaluate Hamiltonian matrixvector products (MVPs). The Hamiltonian is a sum of the kinetic energy operator (KEO) and the potential energy surface (PES). If both are in sum-of-products (SOP) form, then integrals can be decomposed into products of 1-D integrals and MVPs can be evaluated without computing Hamiltonian matrix elements. This is done by evaluating sums sequentially. The idea of summing sequentially has been used, with and without MCTDH, in quantum dynamics for decades. ${ }^{31-34}$ It has surely been used in many fields and its utility is widely recognised. ${ }^{35,36}$ Summing sequentially reduces the cost by orders of magnitude. Many KEOs are a SOP, but PESs fit or interpolated from ab initio points are typically not SOPs (at least not in the coordinates used to solve the Schrödinger equation).

When the PES is not a SOP, there are four good options for evaluating MVPs. First, one can fit a SOP PES to the original PES. This can be done using, e.g., a) the POTFIT algorithm; $; 7-39$ or b) neural networks. ${ }^{40-43}$ Second, one can use an N-mode (or N-body) representation of the PES. ${ }^{44-46}$ The individual terms might be written in SOP form. ${ }^{47}$ Third, one can use some version of the correlation discrete variable representation (CDVR) quadrature scheme. ${ }^{25,48,49}$ Fourth, one can use a collocation-based MCTDH method (C-MCTDH). ${ }^{50,51}$ The first three options facilitate the calculation of potential integrals. The fourth option obviates the need for integrals.

Although viable options, the first three non-SOP options all have disadvantages. The first and second options modify the PES. Refitting may also be costly. Worse, the number of 
terms in the refitted PES might be large, which is undesirable because the cost of a MCTDH calculation with a SOP PES scales linearly with the number of terms. The CDVR, the third option, does not modify the PES. It is a DVR-based quadrature scheme designed to deal with the fact that a simple (time-dependent) DVR basis with as many functions as there are SPFs is so small that the quadrature error is significant. ${ }^{48}$ The quadrature error is reduced by introducing, in addition to the standard (time-dependent) DVR grid a separate "fine" grid for each individual coordinate. To use the CDVR idea with multivariate (nondirect product) SPFs, one needs a multivariate DVR. CDVR methods have been invented that make it possible to use the CDVR idea with multivariate SPFs and are used with both MC- and ML-MCTDH. ${ }^{25,49,52}$ They require neglecting off-diagonal matrix elements in matrices that represent coordinates (or functions of coordinates) in a basis of vectors obtained by nearly simultaneously diagonalising (SD) non-commuting matrices. ${ }^{53}$ The neglect of off-diagonal matrix elements is intrinsic to any SD-DVR approach. It was noted in Ref. 52 that it was not possible to achieve accurate vibrational energies using MC- and ML-MCTDH without introducing weights. The reason that evaluating PES MVPs is challenging, and that the second weakness of MCTDH is limiting, is that is they involve multi-dimensional integrals. Using collocation, there is no need to compute integrals and it is therefore straightforward to use a non-SOP PES; this alleviates the second weakness of standard MCTDH.

To use a collocation method (see, e.g., page 13 in Ref. 54), it is only necessary to evaluate basis functions, derivatives of basis functions, and the PES at collocation points; it is not necessary that it be possible to accurately evaluate integrals with a quadrature whose points are the collocation points. If the number of points is equal to the number of basis functions, then collocation is equivalent to using a variational approach and evaluating all (also the KEO and overlap) matrix elements by quadrature. According to Boys ${ }^{55}$ when all matrix elements are evaluated by quadrature, the error in the computed energy levels decreases as the basis becomes more complete, regardless of the magnitude of the quadrature error. This is why points with which quadrature would be accurate are not required when using collocation. The case for collocation is most compelling when using a good basis and MCTDH provides an excellent basis. Two advantages of C-MCTDH calculations are: 1) there is no need for the CDVR correction (although C-MCTDH also uses 1-D fine grids), ${ }^{48}$ which was introduced to make the CDVR quadrature accurate; 2) there is no need to use weights to make the most important parts of matrices in the SD basis more nearly diagonal. ${ }^{52}$ 
In this paper we solve the C-MCTDH equations with a generalised improved relaxation method. Improved relaxation was originally introduced in Refs. 56,57 and is frequently used with MCTDH to compute energy eigenpairs. ${ }^{58}$ To generalise it, so that it can be used with C-MCTDH, we follow the Petrov-Galerkin approach used to derive the C-MCTDH working equations for solving the time-dependent Schrödinger equation ${ }^{50}$ and derive equations for solving the time-independent Schrödinger equation. We test the mode combination, collocation, improved relaxation approach by calculating the first 36, 80, and 69 vibrational eigenpairs of $\mathrm{CH}_{3}, \mathrm{CH}_{4}$, and $\mathrm{CH}_{3} \mathrm{CN}$, respectively.

\section{COMBINING C-MCTDH AND MODE COMBINATION}

\section{A. C-MCTDH with univariate SPFs}

Before deriving equations that make it possible to combine $\mathrm{MC}$ with $\mathrm{C}-\mathrm{MCTDH}$, we recapitulate, in this subsection, the working equations for a collocation-based MCTDH approach that uses a direct product basis of univariate (1-D) single-particle functions, as in Eq. (1), and a direct product collocation. They are ${ }^{50}$

$$
\mathrm{i} \dot{A}_{i_{1}, \ldots, i_{D}}(t)=\sum_{a_{1}, \ldots, a_{D}} \sum_{j_{1}, \ldots, j_{D}}\left(\left[\mathbf{B}^{(1)}\right]^{-1}\right)_{i_{1}, a_{1}} \ldots\left(\left[\mathbf{B}^{(D)}\right]^{-1}\right)_{i_{D}, a_{D}} \underset{\substack{a_{1}, \ldots, a_{D} \\ j_{1}, \ldots, j_{D}}}{A_{j_{1}, \ldots, j_{D}}}(t)
$$

and

$$
\sum_{i_{k}=1}^{n_{k}} \rho_{i_{k}^{\prime}, i_{k}}^{(k)} \mathrm{i}\left|\dot{\varphi}_{i_{k}}^{(k)}(t)\right\rangle=(\hat{1}-\underbrace{\sum_{j_{k}, a_{k}=1}^{n_{k}}\left|\varphi_{j_{k}}^{(k)}(t)\right\rangle\left(\left[\mathbf{B}^{(k)}\right]^{-1}\right)_{j_{k}, a_{k}}\left\langle r_{a_{k}}^{(k)}(t)\right|}_{=\hat{P}^{(k)}}) \sum_{i_{k}=1}^{n_{k}} \hat{\mathcal{H}}_{i_{k}^{\prime}, i_{k}}^{(k)}\left|\varphi_{i_{k}}^{(k)}(t)\right\rangle .
$$

$r_{a_{k}}^{(k)}(t)$ denotes a collocation point. The matrix elements

$$
B_{a_{k}, i_{k}}^{(k)}=\varphi_{i_{k}}^{(k)}\left(r_{a_{k}}^{(k)}(t), t\right)=\left\langle r_{a_{k}}^{(k)}(t) \mid \varphi_{i_{k}}^{(k)}(t)\right\rangle
$$

are values of the SPFs at collocation points. The other quantities that appear in Eqs. (3) and (4) are the non-Hermitian Hamiltonian matrix

$$
\underset{\substack{a_{1}, \ldots, a_{D} \\ i_{1}, \ldots, i_{D}}}{H}=\left\langle r_{a_{1}}^{(1)}(t)\left|\ldots\left\langle r_{a_{D}}^{(D)}(t)|\hat{H}| \varphi_{i_{1}}^{(1)}(t)\right\rangle \ldots\right| \varphi_{i_{D}}^{(D)}(t)\right\rangle
$$


the mean-field (operator) matrices

$$
\hat{\mathcal{H}}_{i_{k}^{\prime}, i_{k}}^{(k)}=\sum_{i_{1}^{\prime}, \ldots, i_{D}^{\prime}}^{(\neg k)} \sum_{a_{1}, \ldots, a_{D}}^{(\neg k)} \sum_{i_{1}, \ldots, i_{D}}^{(\neg k)} A_{i_{1}^{\prime}, \ldots, i_{D}^{\prime}}^{*} \prod_{j \neq k}\left(\left[\mathbf{B}^{(j)}\right]^{-1}\right)_{i_{j}^{\prime}, a_{j}}\left(\bigotimes_{j \neq k}\left\langle r_{a_{j}}^{(j)}(t)\right|\right) \hat{H}\left(\bigotimes_{j \neq k}\left|\varphi_{i_{j}}^{(j)}(t)\right\rangle\right) A_{i_{1}, \ldots, i_{D}}
$$

and the density matrices

$$
\rho_{i_{k}^{\prime}, i_{k}}^{(k)}=\sum_{j_{1}, \ldots, j_{D}}^{(\neg k)} A_{j_{1}, \ldots, i_{,}^{\prime}, \ldots, j_{D}}^{*} A_{j_{1}, \ldots, i_{k}, \ldots, j_{D}} .
$$

Note that $\neg k$ means sum over all but the $k$-th index and that the form of the 1-D collocation projectors $\hat{P}^{(k)}$ is indicated in Eq. (4) by the underbrace.

To obtain explicit equations for the coefficients $c_{f_{k}, i_{k}}^{(k)}$ we use a fine 1-D collocation grid. Eq. (4) is left-multiplied by $\left\langle\tilde{r}_{\alpha_{k}}^{(k)}\right|$, where $\tilde{r}_{\alpha_{k}}^{(k)}$ is one of $N_{k}$ fine-grid collocation points. They are are chosen to be the Gauss quadrature points corresponding to the $\chi_{f_{k}}^{(k)}\left(x_{k}\right)$ basis functions. One then obtains

$$
\begin{aligned}
\sum_{i_{k}=1}^{n_{k}} \rho_{i_{k}^{\prime}, i_{k}}^{(k)} \mathrm{i} \dot{c}_{f_{k}, i_{k}}^{(k)}(t) & =\sum_{\beta_{k}=1}^{N_{k}} \sum_{i_{k}=1}^{n_{k}} \sum_{f_{k}^{\prime}=1}^{N_{k}}\left(\left[\tilde{\mathbf{B}}^{(k)}\right]^{-1}\right)_{f_{k}, \beta_{k}}\left\langle\tilde{r}_{\beta_{k}}^{(k)}\left|\hat{\mathcal{H}}_{i_{k}^{\prime}, i_{k}}^{(k)}\right| \chi_{f_{k}^{\prime}}^{(k)}\right\rangle c_{f_{k}^{\prime}, i_{k}}^{(k)}(t) \\
& -\sum_{j_{k}=1}^{n_{k}} \sum_{a_{k}=1}^{n_{k}} \sum_{i_{k}=1}^{n_{k}} \sum_{f_{k}^{\prime}=1}^{N_{k}} c_{f_{k}, j_{k}}^{(k)}\left(\left[\mathbf{B}^{(k)}\right]^{-1}\right)_{j_{k}, a_{k}}\left\langle r_{a_{k}}^{(k)}(t)\left|\hat{\mathcal{H}}_{i_{k}^{\prime}, i_{k}}^{(k)}\right| \chi_{f_{k}^{\prime}}^{(k)}\right\rangle c_{f_{k}^{\prime}, i_{k}}^{(k)}(t),
\end{aligned}
$$

where

$$
\tilde{B}_{\alpha_{k}, f_{k}}^{(k)}=\chi_{f_{k}}^{(k)}\left(\tilde{r}_{\alpha_{k}}^{(k)}\right)
$$

Throughout the paper, $i$ and $j$ label SPFs; $a$ and $b$ label points on the coarse product grid; and $\alpha$ and $\beta$ label points on the fine Gauss grid; and $f$ labels the primitive $\chi_{f_{k}}^{(k)}$ functions used to compute the SPFs. Both CDVR and C-MCTDH require fine 1-D grids for each coordinate and a coarse TD-DVR grid (there is also a pruned C-MCTDH ${ }^{51}$ in which the coarse grid is not a direct product), however, in C-MCTDH the fine grids are introduced only to solve for the SPFs. In C-MCTDH, because there is no quadrature, there is no need to correct matrix elements when computing the $A$ coefficients. 


\section{B. C-MCTDH with multivariate SPFs}

If in Eq. (2), one uses multivariate single-particle functions, i.e., one combines $d_{\ell}$ physical coordinates into logical coordinates ${ }^{1,19}$

$$
q_{\ell}=(\underbrace{x_{k_{1, \ell}, \ldots, x_{k_{\ell}, \ell}}}_{d_{\ell} \text { physical coordinates }}) \text { for } \ell=1 \ldots D^{\prime},
$$

one obtains the mode combination MCTDH wavefunction

$$
\Psi\left(q_{1}, \ldots, q_{D^{\prime}}, t\right)=\sum_{i_{1}=1}^{n_{1}^{\prime}} \ldots \sum_{i_{D^{\prime}}=1}^{n_{D^{\prime}}^{\prime}} A_{i_{1}, \ldots, i_{D^{\prime}}}(t) \varphi_{i_{1}}^{(1)}\left(q_{1}, t\right) \ldots \varphi_{i_{D^{\prime}}}^{\left(D^{\prime}\right)}\left(q_{D^{\prime}}, t\right)
$$

with

$$
\begin{aligned}
\varphi_{i_{\ell}}^{(\ell)}\left(q_{\ell}, t\right)= & \varphi_{i_{\ell}}^{(\ell)}\left(x_{k_{1, \ell}}, \ldots, x_{k_{d_{\ell}, \ell}}, t\right)=\sum_{f_{1}=1}^{N_{k_{1}, \ell}} \ldots \sum_{f_{d_{\ell}}=1}^{N_{k_{d_{\ell}, \ell}}} c_{f_{1}, \ldots, f_{d_{\ell}} ; i_{\ell}}^{(\ell)}(t) \chi_{f_{1}}^{\left(k_{1, \ell}\right)}\left(x_{k_{1, \ell}}\right) \ldots \chi_{f_{d_{\ell}}}^{\left(k_{d_{\ell}, \ell}\right)}\left(x_{k_{d_{\ell}, \ell}}\right) \\
& \forall \ell=1 \ldots D^{\prime} .
\end{aligned}
$$

Since coupling between physical coordinates that constitute a logical coordinate is incorporated in the multi-dimensional SPFs, often significantly fewer multivariate SPFs are required in Eq. (12) than in Eq. (1), i.e., $\prod_{\ell=1}^{D^{\prime}} n_{\ell}^{\prime}<\prod_{k=1}^{D} n_{k}$. In practice, it seems to be most efficient to combine between 2 and 4 physical coordinates. The more coordinates are combined, the more costly it is to solve Eq. (13) (however pruning can also be used to reduce the cost of calculating multivariate SPFs). ${ }^{30}$

It is simple to generalise the C-MCTDH equations so that they can be used with multivariate SPFs. The MC-C-MCTDH equations are:

$$
\mathrm{i} \dot{A}_{i_{1}, \ldots, i_{D^{\prime}}}(t)=\sum_{a_{1}, \ldots, a_{D^{\prime}}} \sum_{j_{1}, \ldots, j_{D^{\prime}}}\left(\left[\mathbf{B}^{(1)}\right]^{-1}\right)_{i_{1}, a_{1}} \ldots\left(\left[\mathbf{B}^{\left(D^{\prime}\right)}\right]^{-1}\right)_{\substack{i_{D^{\prime}}, a_{D^{\prime}} \\ H a_{1}, \ldots, a_{D^{\prime}}, \ldots, j_{D^{\prime}}}} A_{j_{1}, \ldots, j_{D^{\prime}}}(t),
$$

and

$$
\sum_{i_{\ell}=1}^{n_{\ell}^{\prime}} \rho_{i_{\ell}^{\prime}, i_{\ell}}^{(\ell)} \mathrm{i}\left|\dot{\varphi}_{i_{\ell}}^{(\ell)}(t)\right\rangle=(\hat{1}-\underbrace{\sum_{j_{\ell}, a_{\ell}=1}^{n_{\ell}^{\prime}}\left|\varphi_{j_{\ell}}^{(\ell)}(t)\right\rangle\left(\left[\mathbf{B}^{(\ell)}\right]^{-1}\right)_{j_{\ell}, a_{\ell}}\left\langle r_{a_{\ell}}^{(\ell)}(t)\right|}_{=\hat{P}^{(\ell)}}) \sum_{i_{\ell}=1}^{n_{\ell}^{\prime}} \hat{\mathcal{H}}_{i_{\ell}^{\prime}, i_{\ell}}^{(\ell)}\left|\varphi_{i_{\ell}}^{(\ell)}(t)\right\rangle .
$$


Analogous to Eq. (9), explicit mode-combination equations for the coefficients $c_{\mathbf{f}_{\ell}, i_{\ell}}^{(\ell)}$ are given by

$$
\begin{aligned}
& \sum_{i_{\ell}=1}^{n_{\ell}^{\prime}} \rho_{i_{\ell}^{\prime}, i_{\ell}}^{(\ell)} \mathrm{i} \dot{c}_{\mathbf{f}_{\ell}, i_{\ell}}^{(\ell)}(t) \\
& \left.=\sum_{\beta_{1}=1}^{N_{k_{1}, \ell}} \ldots \sum_{\beta_{d_{\ell}}=1}^{N_{k_{d_{\ell}, \ell}}} \sum_{i_{\ell}=1}^{n_{\ell}^{\prime}} \sum_{f_{1}^{\prime}=1}^{N_{k_{1, \ell}}} \ldots \sum_{f_{d_{\ell}}^{\prime}=1}^{N_{k_{d_{\ell}, \ell}}}\left(\left[\tilde{\mathbf{B}}^{\left(k_{1, \ell}\right)}\right]^{-1} \otimes \ldots \otimes\left[\tilde{\mathbf{B}}^{\left(k_{d_{\ell}}, \ell\right.}\right)\right]^{-1}\right)_{\mathbf{f}_{\ell}, \boldsymbol{\beta}_{\ell}} \\
& \left\langle\tilde{r}_{\beta_{1}}^{\left(k_{1, \ell}\right)}\left|\ldots\left\langle\tilde{r}_{\beta_{d_{\ell}}}^{\left(k_{d_{\ell}, \ell}\right)}\left|\hat{\mathcal{H}}_{i_{\ell}^{\prime}, i_{\ell}}^{(\ell)}\right| \chi_{f_{1}^{\prime}}^{\left(k_{1, \ell}\right)}\right\rangle \ldots\right| \chi_{f_{d_{\ell}}^{\prime}}^{\left(k_{d_{\ell}, \ell}\right)}\right\rangle c_{\mathbf{f}_{\ell}^{\prime}, i_{\ell}}^{(\ell)}(t) \\
& -\sum_{j_{\ell}=1}^{n_{\ell}^{\prime}} \sum_{a_{\ell}=1}^{n_{\ell}^{\prime}} \sum_{i_{\ell}=1}^{n_{\ell}^{\prime}} \sum_{f_{1}^{\prime}=1}^{N_{k_{1, \ell}}} \ldots \sum_{f_{d_{\ell}}^{\prime}=1}^{N_{k_{d_{\ell}, \ell}}} c_{\mathbf{f}_{\ell}, j_{\ell}}^{(\ell)}\left(\left[\mathbf{B}^{(\ell)}\right]^{-1}\right)_{j_{\ell}, a_{\ell}}\left\langle r_{a_{\ell}}^{(\ell)}(t)\left|\hat{\mathcal{H}}_{i_{\ell}^{\prime}, i_{\ell}}^{(\ell)}\right| \chi_{\left.f_{1}^{\prime}, l^{\prime}\right)}^{\left(k_{1}\right)} \ldots \mid \chi_{f_{d_{\ell}}^{\prime}}^{\left(k_{d_{\ell}, \ell}\right)}\right\rangle c_{\mathbf{f}_{\ell}^{\prime}, i_{\ell}}^{(\ell)}(t) .
\end{aligned}
$$

Note that each each fine point label $\alpha_{k}$ has been replaced with a composite index $\boldsymbol{\alpha}_{\ell}$ with $d_{\ell}$ components and each primitive basis label $f_{k}$ has been replaced with a composite index $\boldsymbol{f}_{\ell}$ with $d_{\ell}$ components. For a particular $\ell, \mathbf{B}^{(\ell)}$ is an $n_{\ell}^{\prime} \times n_{\ell}^{\prime}$ matrix whose elements are $d_{\ell}$-dimensional functions evaluated at points in $d_{\ell^{-}}$dimensional space. 


\section{CHOOSING COLLOCATION POINTS}

It is well and good to write MC collocation equations (14), (15), and (16), but to solve them, one must choose collocation points. The accuracy of the solutions depends on which points are used. Even without MC, this is an issue. When using a collocation method, one finds an interpolant that satisfies a given differential equation exactly (in the space spanned by the basis functions) at a certain set of points. It is best to choose interpolation/collocation points to minimise the so-called Lebesgue constant, see, e.g., Ref. 59. The Lebesgue constant increases as the number of points (and basis functions) increases. In a polynomial basis, the Lebesgue constant of the best (Chebyshev) points increases only logarithmically. ${ }^{59}$ The Lebesgue constant bounds the interpolation error of a function; the lower the Lebesgue constant, the closer the interpolant is to the best approximation of the function within the space of basis functions. One way to find points that minimise the Lebesgue constant is to maximise the determinant of $\mathbf{B}$, the matrix whose elements are values of the basis functions at the collocation points. Such points are called Fekete points. ${ }^{60}$

When basis functions are 1-D weighted orthogonal polynomials, it is clear that Gauss quadrature (DVR) points are a good choice, because they ensure that the determinant of B is not small. Correspondingly, it is natural when using MCTDH, without MC, to use as collocation points, TD-DVR points which are the eigenvalues of matrices whose elements are

$$
X_{i_{k}, j_{k}}^{(k)}=\sum_{i_{k}^{\prime}, j_{k}^{\prime}=1}^{n_{k}}\left(\left[\mathbf{S}^{(k)}\right]^{-1 / 2}\right)_{i_{k}, i_{k}^{\prime}}\left\langle\varphi_{i_{k}^{\prime}}^{(k)}(t)\left|\hat{x}_{k}\right| \varphi_{j_{k}^{\prime}}^{(k)}(t)\right\rangle,\left(\left[\mathbf{S}^{(k)}\right]^{-1 / 2}\right)_{j_{k}^{\prime}, j_{k}} .
$$

$\mathbf{S}^{(k)}=\left(\mathbf{c}^{(k)}\right)^{\dagger} \mathbf{c}^{(k)}$ is the overlap matrix of the, in general, non-orthonormal SPFs, and $\left\langle\varphi_{i_{k}^{\prime}}^{(k)}(t)\left|\hat{x}_{k}\right| \varphi_{j_{k}}^{(k)}(t)\right\rangle$ is computed with the Gauss quadrature that corresponds to $\chi_{f_{k}}^{(k)}\left(x_{k}\right)$. This is what we did in Ref. 50 .

For 1-D basis functions, Leja points also maximise $\operatorname{det}(\mathbf{B}) \cdot{ }^{61-64}$ The associated Lebesgue constant grows at most subexponentially with the number of interpolation points. ${ }^{62}$ Originally, Leja points were employed in conjunction with polynomial bases, ${ }^{64-67}$ but there also exist recipes for obtaining Leja points for general basis functions. ${ }^{51,68-70}$. Leja points form a sequence, i.e., the $k$ Leja points obtained using the basis functions $\left\{\varphi_{1}, \ldots, \varphi_{k}\right\}$ are a subset of the $k+1$ points generated when using $\left\{\varphi_{1}, \ldots, \varphi_{k}, \varphi_{k+1}\right\}$ as the basis. To determine the 
$x_{k+1}$ Leja point in the sequence one has to find the maximum

$$
x_{k+1}=\underset{x}{\arg \max }\left|\varphi_{k+1}(x)-\left[U^{k}\left(x_{1}, x_{2}, \ldots, x_{k}\right) \varphi_{k+1}\right](x)\right|,
$$

where $U^{k}\left(x_{1}, x_{2}, \ldots, x_{k}\right)\left[\varphi_{k+1}(x)\right]$ is the interpolant of $\varphi_{k+1}(x)$ made with $k$ basis functions and points. Recently, we used Leja points in a C-MCTDH approach that employs nondirect (pruned) SPF bases and a Smolyak collocation grid. ${ }^{51}$

To use MC with collocation, we need collocation points $\mathbf{r}_{a_{\ell}}^{(\ell)}=\left(r_{a_{\ell}}^{\left(k_{1, \ell}\right)}, \ldots, r_{a_{\ell}}^{\left(k_{d_{\ell}, \ell}\right)}\right)$ for multi-dimensional coordinates $q_{\ell}=\left(x_{k_{1, \ell}, \ldots, x_{k_{\ell}, \ell}}\right)$. How does one determine DVR points and Leja points with mode-combined basis functions, when the basis functions are multivariate? The generalisation to multi-D Leja points is easy. The Leja recipe of Eq. (18) can be used even when the basis functions are multi-variate. The only difficulty is the maximisation problem is now multi-dimensional. Finding points from a DVR-type recipe is not simple if the multi-D basis is not a direct product. This is because matrices representing $x_{p}, p=k_{1, \ell}, \ldots, k_{d_{\ell}, \ell}$, do not commute. For multivariate SPFs, we shall use in this paper collocation points that are SD-DVR points. ${ }^{53}$ The same points are also used in ML-CDVR ${ }^{52}$ , but note that to make a CDVR one needs not only points but also the corresponding DVR functions. For one logical coordinate, SD-DVR points are obtained by building $d_{\ell}$ matrices like $\mathbf{X}$ in Eq. (17), for each $x_{k_{i, \ell}}$, and finding a single transformation matrix that nearly simultaneously diagonalises all of them. Doing this for all the logical coordinates gives $D^{\prime}$ sets of $d_{\ell}$-dimensional collocation points. The SD is done using the ideas of Refs. 49,53. Because the matrices do not commute, the transformed matrices are not perfectly diagonal. Whenever an SD-DVR is used to do quadrature, the accuracy of the results is limited by the need to neglect off-diagonal matrix elements that are small but nonzero. This approximation is not necessary when using collocation. The deficiencies of SD-DVR therefore do not limit the accuracy. The accuracy of vibrational levels is limited by neglecting off-diagonal elements when using the original SD-DVR ${ }^{53}$ and when using the CDVR based on it, tested in Ref. 52. The SD-DVR CDVR is accurate enough for photodissociation. ${ }^{25,49}$ The generalisation to multi-D Leja points is easier. The Leja recipe of Eq. (18) can be used even when the basis functions are multi-variate. The only difficulty is the maximisation problem is now multi-dimensional.

Because the SPFs evolve in time, the SD-DVR points and the Leja points are also timedependent. In previous papers, we showed that it is also possible to use C-MCTDH with 
time-independent points. The time independent points are the SD-DVR or Leja points determined from an initial set of SPFs. In this paper we show that the same idea works with MC. Though results obtained with time-dependent points will, in general, be more accurate than those obtained with time-independent points, time-independent points have the advantage that the PES need only be evaluated at the collocation points once. ${ }^{50}$ This is desirable because the repeated evaluation of the PES is typically one of the main bottlenecks of MCTDH/CDVR calculations. The cost of evaluating the PES is larger when one uses $\mathrm{MC}$ because the grids for the logical coordinate are much larger than the 1-D grids for the physical coordinates.

\section{USING IMPROVED RELAXATION WITH C-MCTDH}

In Ref. 50, we used a block relaxation method ${ }^{71}$ to solve C-MCTDH equations to compute vibrational energy levels. Relaxation is a standard trick that enables one to extract solutions of the time-independent Schrödinger equation by solving the time-dependent Schrödinger equation, which MCTDH is designed to do. It is now more common to use "improved relaxation" when computing a spectrum with MCTDH. ${ }^{56,72}$ Improved relaxation equations were first derived from a time-independent variational principle. In this section we derive equations for using improved relaxation with C-MCTDH. In subsection IV A, we rederive the standard improved relaxation equations, not with a variational principle, but with an equivalent Galerkin approach. In subsection IVB, we derive new C-MCTDH improved relaxation equations, using the Petrov-Galerkin approach of Ref. 50. The derivation of subsection IV A is included because it paves the way for the derivation of subsection IV B.

\section{A. Rederiving the improved relaxation equations using a Galerkin approach}

We follow Sec. IV of Ref. 50 and project the residual $(\hat{H}-E)|\Psi\rangle$. To determine the $A$ coefficients and the SPFs for coordinate $k$ (which could be either a physical or a logical coordinate), respectively, we demand that

$$
\bigotimes_{k=1}^{D}\left\langle\varphi_{i_{k}}^{(k)} \mid(\hat{H}-E) \Psi\right\rangle=0
$$


and

$$
\bigotimes_{\ell \neq k}\left\langle\varphi_{i_{\ell}}^{(\ell)} \mid(\hat{H}-E) \Psi\right\rangle=0
$$

Rearranging Eq. (19), one immediately obtains

$$
\sum_{i_{1}, \ldots, i_{D}}\left\langle\varphi_{j_{1}}^{(1)}\left|\ldots\left\langle\varphi_{j_{D}}^{(D)}|\hat{H}| \varphi_{i_{1}}^{(1)}\right\rangle \ldots\right| \varphi_{i_{D}}^{(D)}\right\rangle A_{i_{1}, \ldots, i_{D}}=E A_{j_{1}, \ldots, j_{D}}
$$

Thus, energies and $A$ coefficients are determined by solving the eigenvalue problem in Eq. (21) for the matrix representing the Hamiltonian in the product SPF basis (here, we assume that the SPFs are orthonormal to avoid an overlap matrix on the right-hand side, but it is also possible to deal with an overlap matrix that is not an identity matrix).

The derivation of the equation for the SPFs is more involved. ¿From Eq. (20) one first finds

$$
\sum_{i_{1}, \ldots, i_{D}}\left(\bigotimes_{\ell \neq k}\left\langle\varphi_{j_{\ell}}^{(\ell)}\right|\right) \hat{H}\left(\bigotimes_{\ell \neq k}\left|\varphi_{i_{\ell}}^{(\ell)}\right\rangle\right) A_{i_{1}, \ldots, i_{D}}\left|\varphi_{i_{k}}^{(k)}\right\rangle=E \sum_{i_{k}} A_{j_{1}, \ldots, i_{k}, \ldots, j_{D}}\left|\varphi_{i_{k}}^{(k)}\right\rangle .
$$

Replacing $E A_{j_{1}, \ldots, i_{k}, \ldots, j_{D}}$ on the RHS of Eq. (22) with the LHS of Eq. (21) and multiplying both sides of the resulting equation from the left with $\sum_{j_{1}, \ldots, j_{D}}^{(\neg k)} A_{j_{1}, \ldots, i_{k}^{\prime}, \ldots, j_{D}}$ yields

$$
\begin{aligned}
& \sum_{i_{k}} \sum_{j_{1}, \ldots, j_{D}}^{(\neg k)} \sum_{i_{1}, \ldots, i_{D}}^{(\neg k)} A_{j_{1}, \ldots, i_{k}^{\prime}, \ldots, j_{D}}\left(\bigotimes_{\ell \neq k}\left\langle\varphi_{j_{\ell}}^{(\ell)}\right|\right) \hat{H}\left(\bigotimes_{\ell \neq k}\left|\varphi_{i_{\ell}}^{(\ell)}\right\rangle\right) A_{i_{1}, \ldots, i_{k}, \ldots, i_{D}}\left|\varphi_{i_{k}}^{(k)}\right\rangle \\
& =\sum_{i_{k}} \sum_{j_{1}, \ldots, j_{D}}^{(k)} \sum_{j_{1}^{\prime}, \ldots, j_{D}^{\prime}} A_{j_{1}, \ldots, i_{k}^{\prime}, \ldots, j_{D}}\left\langle\varphi_{j_{1}}^{(1)}\left|\ldots\left\langle\varphi_{i_{k}}^{(k)}\left|\ldots\left\langle\varphi_{j_{D}}^{(D)}|\hat{H}| \varphi_{j_{1}^{\prime}}^{(1)}\right\rangle \ldots\right| \varphi_{j_{k}^{\prime}}^{(k)}\right\rangle \ldots\right| \varphi_{j_{D}^{\prime}}^{(D)}\right\rangle A_{j_{1}^{\prime}, \ldots, j_{k}^{\prime}, \ldots, j_{D}^{\prime}}\left|\varphi_{i_{k}}^{(k)}\right\rangle \\
& =\left(\sum_{i_{k}}\left|\varphi_{i_{k}}^{(k)}\right\rangle\left\langle\varphi_{i_{k}}^{(k)}\right|\right) \sum_{i_{k}} \sum_{j_{1}, \ldots, j_{D}}^{(\neg k)} \sum_{i_{1}, \ldots, i_{D}}^{(\neg k)} A_{j_{1}, \ldots, i_{k}^{\prime}, \ldots, j_{D}}\left(\bigotimes_{\ell \neq k}\left\langle\varphi_{j_{\ell}}^{(\ell)}\right|\right) \hat{H}\left(\bigotimes_{\ell \neq k}\left|\varphi_{i_{\ell}}^{(\ell)}\right\rangle\right) A_{i_{1}, \ldots, i_{k}, \ldots, i_{D}}\left|\varphi_{i_{k}}^{(k)}\right\rangle .
\end{aligned}
$$

Identifying the projector

$$
\hat{P}^{(k)}=\sum_{i_{k}}\left|\varphi_{i_{k}}^{(k)}\right\rangle\left\langle\varphi_{i_{k}}^{(k)}\right|
$$

and the mean-field matrices

$$
\hat{\mathcal{H}}_{i_{k}^{\prime}, i_{k}}^{(k)}=\sum_{j_{1}, \ldots, j_{D}}^{(\neg k)} \sum_{i_{1}, \ldots, i_{D}}^{(\neg k)} A_{j_{1}, \ldots, i_{k}^{\prime}, \ldots, j_{D}}\left(\bigotimes_{\ell \neq k}\left\langle\varphi_{j_{\ell}}^{(\ell)}\right|\right) \hat{H}\left(\bigotimes_{\ell \neq k}\left|\varphi_{i_{\ell}}^{(\ell)}\right\rangle\right) A_{i_{1}, \ldots, i_{k}, \ldots, i_{D}},
$$


one can write Eq. (23) in the more compact form

$$
\sum_{i_{k}} \hat{\mathcal{H}}_{i_{k}^{\prime}, i_{k}}^{(k)}\left|\varphi_{i_{k}}^{(k)}\right\rangle=\hat{P}^{(k)} \sum_{i_{k}} \hat{\mathcal{H}}_{i_{k}^{\prime}, i_{k}}^{(k)}\left|\varphi_{i_{k}}^{(k)}\right\rangle
$$

or

$$
\left(\hat{1}-\hat{P}^{(k)}\right) \sum_{i_{k}} \hat{\mathcal{H}}_{i_{k}^{\prime}, i_{k}}^{(k)}\left|\varphi_{i_{k}}^{(k)}\right\rangle=0
$$

Equations (21) and (26) are exactly the same as those presented in Ref. 56 and derived from a time-independent variational principle. To solve Eq. (26), we follow Ref. 56 and multiply both sides on the left with $\sum_{i_{k}^{\prime}}\left(\left[\boldsymbol{\rho}^{(k)}\right]^{-1}\right)_{j_{k}, i_{k}^{\prime}}$ and determine $\left|\varphi_{i_{k}}^{(k)}\right\rangle$ by solving the corresponding time-dependent Schrödinger equation in imaginary time:

$$
\left|\dot{\varphi}_{j_{k}}^{(k)}\right\rangle=-\left(\hat{1}-\hat{P}^{(k)}\right) \sum_{i_{k}^{\prime}, i_{k}}\left(\left[\boldsymbol{\rho}^{(k)}\right]^{-1}\right)_{j_{k}, i_{k}^{\prime}} \hat{\mathcal{H}}_{i_{k}^{\prime}, i_{k}}^{(k)}\left|\varphi_{i_{k}}^{(k)}\right\rangle=0
$$

where $\left|\dot{\varphi}_{j_{k}}^{(k)}\right\rangle$ is the derivative of a SPF with respect to $\tau=\mathrm{i}$. SPFs are therefore obtained by relaxation and energies and $A$ coefficients are generated by solving the eigenvalue problem of Eq. (21). In practice, an iterative (subspace) method ${ }^{73}$ is used for the latter task to avoid the storage and manipulation of huge matrices, typically a Lanczos/Arnoldi or Davidson ${ }^{74}$ eigensolver.

Eq. (27) and Eq. (21) enable one to use MCTDH to compute a single eigenpair. It is straightforward to use them to compute the ground state. It is also possible to compute excited states, one at a time, ${ }^{56}$ but is more efficient to use a state-averaged MCTDH ansatz to simultaneously calculate several eigenstates. ${ }^{57,71} s>1$ wavefunctions are represented in a single set of SPFs and the energies and coefficients in Eq. (21) have an additional index s. In the definition of the mean-field, Eq. (25), and density matrices, Eq. (8), an additional sum over the $s$ states is required, ${ }^{71}$ but the SPF equations have the same form.

\section{B. Deriving C-MCTDH improved relaxation equations}

Following the derivation of subsection IV A, it is possible to obtain the equations one must solve to use improved relaxation with C-MCTDH. As in Ref. 50, the C-MCTDH equations can be obtained by replacing the bras in Eqs. (19) and (20) by $\left\langle r_{a_{k}}^{(k)}\right|$. The eigenvalue 
problem to be solved to obtain energies and $A$-coefficients is

$$
\sum_{a_{1}, \ldots, a_{D}} \sum_{i_{1}, \ldots, i_{D}}\left(\left[\mathbf{B}^{(1)}\right]^{-1}\right)_{j_{1}, a_{1}} \ldots\left(\left[\mathbf{B}^{(D)}\right]^{-1}\right)_{j_{D}, a_{D}}\left\langle r_{a_{1}}^{(1)}\left|\ldots\left\langle r_{a_{D}}^{(D)}|\hat{H}| \varphi_{i_{1}}^{(1)}\right\rangle \ldots\right| \varphi_{i_{D}}^{(D)}\right\rangle A_{i_{1}, \ldots, i_{D}}=E A_{j_{1}, \ldots, j_{D}}
$$

where the $\mathbf{B}^{(k)}$ matrices are given in Eq. (5). The differential equation for the SPFs has the same form as Eq. (27), but the mean-field matrices are those in Eq. (7) and the projector is that in Eq. (4). To numerically solve Eq. (27), we left-multiply with $\left\langle\tilde{r}_{\alpha_{k}}^{(k)}\right|$, where $\tilde{r}_{\alpha_{k}}^{(k)}$ is a fine-grid collocation point, cf. Eq. (9). When MC is used one must change $D$ to $D^{\prime}$ and use multi-dimensional points and SPFs.

A state-averaged improved relaxation method can be used with C-MCTDH to efficiently calculate many energy levels. Because $\mathbf{B}^{-1} \mathbf{H}$ in Eq. (28) is not Hermitian, where

$$
\mathbf{B}^{-1}=\left[\mathbf{B}^{(1)}\right]^{-1} \otimes\left[\mathbf{B}^{(2)}\right]^{-1} \otimes \ldots\left[\mathbf{B}^{(D)}\right]^{-1},
$$

it is not possible to use the Davidson or Lanczos algorithms to compute the required eigenvalues. Instead, one must use either an extended version of the Davidson algorithm ${ }^{74}$ or an Arnoldi procedure. Since we are interested in the lowest $\sim 100$ eigenstates in this work, we opt for the latter and use the implicitly restarted Arnoldi algorithm that is implemented in the ARPACK package. ${ }^{75}$ The eigenvalues of $\mathbf{B}^{-1} \mathbf{H}$ are not upper bounds for the exact energies and if particularly poor points are chosen they can even be complex. To solve Eqs. (28) and (27) simultaneously, we employ the constant mean-field integrator ${ }^{76}$ CMF2 described in Ref. 77.

\section{VIBRATIONAL ENERGY LEVELS OF $\mathrm{CH}_{3}, \mathrm{CH}_{4}, \mathrm{AND} \mathrm{CH}_{3} \mathrm{CN}$}

To test the combination of C-MCTDH with mode combination and improved relaxation, we calculate the lowest vibrational energy eigenpairs of three molecules: the methyl radical (6-D), methane (9-D), and acetonitrile (12-D). We use a quartic force field PES for $\mathrm{CH}_{3} \mathrm{CN}^{78}$ that is based on force constants reported in Ref. 79. Due to the SOP structure of the PES, we can compare the C-MCTDH results with accurate vibrational energies ${ }^{28}$ calculated with a P-MCTDH approach in which all matrix elements are calculated exactly. The PESs we use for $\mathrm{CH}_{3}{ }^{80}$ and $\mathrm{CH}_{4}{ }^{81}$ are not in SOP form and we compare the C-MCTDH energies with those reported in Refs. 80 and 81 in which quadrature is used to evaluate PES matrix elements. 


\section{A. Coordinates and $\mathrm{KEO}$ for $\mathrm{CH}_{3}$ and $\mathrm{CH}_{4}$}

The coordinates we use for $\mathrm{CH}_{3}$ and $\mathrm{CH}_{4}$ are lengths and angles associated with three and four Radau vectors, ${ }^{82}$ respectively. For $\mathrm{CH}_{3}$, we use the (weighted with the $\mathrm{H}$ atom mass) lengths $\left(r_{1}, r_{2}, r_{3}\right)$, the angle $\theta$ between $\mathbf{r}_{1}$ and $\mathbf{r}_{2}$, and stereographic coordinates ${ }^{83,84}$ $\left(s_{3}, t_{3}\right)$ that specify the orientation of $\mathbf{r}_{3}$ with respect to the first two Radau vectors. For $\mathrm{CH}_{4}$, we add to those coordinates the length $r_{4}$ and the stereographic coordinates $\left(s_{4}, t_{4}\right)$ that determine the length and orientation of the fourth Radau vector $\mathbf{r}_{4}$. A general rovibrational KEO for molecular systems that are described by $\left(r_{1}, r_{2}, \theta\right)$, sets of $\left(r_{3}, s_{3}, t_{3}\right)$, $\left(r_{4}, s_{4}, t_{4}\right), \ldots$, and three body-fixed rotational angular momentum operators has been derived and implemented in Ref. 84. ¿From the general form reported there, it is straightforward to extract the KEOs for the vibrational motion of $\mathrm{CH}_{3}$ and $\mathrm{CH}_{4}$; the explicit form of the vibrational $\mathrm{KEO}$ for $\mathrm{CH}_{3}$ has been given recently. ${ }^{50}$

\section{B. Coordinates and $\mathrm{KEO}$ for $\mathrm{CH}_{3} \mathrm{CN}$}

Dimensionless normal coordinates $q_{k}$ are used for $\mathrm{CH}_{3} \mathrm{CN}$. The Hamiltonian is

$$
\hat{H}=-\frac{1}{2} \sum_{k=1}^{12} \omega_{k} \frac{\partial^{2}}{\partial q_{k}^{2}}+V(\mathbf{q}),
$$

where $V(\mathbf{q})$ is the quartic force field potential of Ref. 78. The frequencies $\omega_{k}\left(\right.$ in $\mathrm{cm}^{-1}$ ) and the respective $C_{3 v}$ symmetry labels are $\omega_{1}=3065\left(\mathrm{~A}_{1}\right), \omega_{2}=2297\left(\mathrm{~A}_{1}\right), \omega_{3}=1413\left(\mathrm{~A}_{1}\right)$, $\omega_{4}=920\left(\mathrm{~A}_{1}\right), \omega_{5,6}=3149(\mathrm{E}), \omega_{7,8}=1487(\mathrm{E}), \omega_{9,10}=1061(\mathrm{E}), \omega_{11,12}=361(\mathrm{E})$.

\section{Combined modes and basis set sizes}

Figs. 1 (a), (b), and (c) show the mode combination schemes used for $\mathrm{CH}_{3}, \mathrm{CH}_{4}$, and $\mathrm{CH}_{3} \mathrm{CN}$, respectively. Similar tree diagrams (but with more levels) are frequently used for ML-MCTDH calculations ${ }^{25}$ and have also been used in the hierarchical variant ${ }^{85}$ of the Reduced Rank Block Power Method ${ }^{86}$. Each node (black dot) in a tree denotes a set of coefficients; the top node represents the $A$ tensor and the coefficients representing the SPFs are indicated by the nodes on lower layers. The number of multi-dimensional single-particle functions employed when computing the $A$ tensor is shown next to the line connecting the 
FIG. 1: The MC-MCTDH wavefunction representations used to calculate vibrational eigenpairs of $\mathrm{CH}_{3}, \mathrm{CH}_{4}$, and $\mathrm{CH}_{3} \mathrm{CN}$, respectively. $\mathrm{HO}$ denotes a harmonic oscillator basis, LP a Legendre polynomial basis, and PW a plane wave basis. For $\mathrm{CH}_{3} \mathrm{CN}$, all primitive basis functions are HOs. The modes $q_{1} \ldots q_{4}$ are non-degenerate, whereas $\left(q_{5}, q_{6}\right) \ldots\left(q_{11}, q_{12}\right)$ are doubly degenerate.

top node and other nodes. The primitive basis set sizes are given at the bottom of the tree with the physical coordinates. The type of primitive basis functions used for each of the coordinates is given with the abbreviations $\mathrm{HO}=$ harmonic oscillator basis, $\mathrm{LP}=$ Legendre polynomial basis, and PW = plane wave basis. The vertical line on the top node 
indicates that the state-averaged MCTDH approach $^{71}$ is employed to calculate the number of wavefunctions shown next to the line.

It is of course best to combine coordinates that are strongly coupled. In $\mathrm{CH}_{3} \mathrm{CN}$, we combine degenerate coordinates with the non-degenerate coordinate whose frequency is closest to the frequency of the degenerate pair. In $\mathrm{CH}_{3}$ and $\mathrm{CH}_{4}$, we combine the stretching modes together and combine $\left(s_{i}, t_{i}\right)$ stereographical coordinates for each hydrogen atom. $\left(s_{i}, t_{i}\right)$ should be strongly correlated as the natural motions of the hydrogens in $\mathrm{CH}_{3}$ and $\mathrm{CH}_{4}$ alter the values of the $s$ and $t$ coordinates simultaneously.

\section{Collocation points}

For the calculations of vibrational levels of $\mathrm{CH}_{3} \mathrm{CN}$ and $\mathrm{CH}_{3}$, we use SD-DVR collocation points for all the coordinates. In $\mathrm{CH}_{4}$, we use SD-DVR points for the stretching coordinates and for $\theta$. For the stereographical coordinates, we use 2-D Leja points. We use Leja points because they significantly reduce the condition numbers of the $\left(s_{i}, t_{i}\right) \mathbf{B}^{(k)}$ matrices that must be inverted to solve the C-MCTDH equations, see Eq. (3). We have confirmed that $\mathrm{CH}_{3}$ energies computed using Leja points for the stereographical coordinates agree well with those calculated with SD-DVR points, provided the basis is large enough.

\section{E. Results}

As a first test, we calculate the lowest 69 vibrational energy levels of $\mathrm{CH}_{3} \mathrm{CN}$ with C$\mathrm{MCTDH}$ and the combined modes indicated in Figure 1 (c) using improved relaxation. The points evolve with the SPFs. Because the corresponding PES is given in SOP form, we can compare the energies to the accurate results previously obtained with P-MCTDH, which requires that it be possible to evaluate all matrix elements exactly. ${ }^{28}$ Errors for all levels are less than $0.1 \mathrm{~cm}^{-1}$ with the majority of levels deviating from the reference values by less than $0.01 \mathrm{~cm}^{-1}$.

As a second test, we calculate the lowest 36 vibrational energy levels of $\mathrm{CH}_{3}$ with the bases of Fig. 1 (b). They have errors similar to those of $\mathrm{CH}_{3} \mathrm{CN}$. Unlike $\mathrm{CH}_{3} \mathrm{CN}$, the PES is not a SOP of factors that depend on the coordinates in which we solve the Schrödinger equation. For $\mathrm{CH}_{3}$, we have confirmed that energies computed with time-independent points 
are about equally accurate. To do this, we employ the strategy described in Ref. 50 and do a set of calculations with bases $B_{1}, B_{2}, \ldots$ of increasing size and use points determined with $\mathrm{B}_{n}$ to do the calculation with $\mathrm{B}_{n+1}$. The final basis is the one indicated in Fig. 1 (b). Even when we do not use time-independent points, a series of calculations with different bases is done to check convergence, thus choosing to use the points from the calculation with $\mathrm{B}_{n}$ to do the calculation with $\mathrm{B}_{n+1}$ does not significantly increase the cost. Though the energies computed with time-independent points have slightly larger errors, they are still all in the sub- $\mathrm{cm}^{-1}$ range.

In Tab. I, we report some of the first 80 (up to and including the octad) vibrational energies of $\mathrm{CH}_{4}$. Errors for all the levels (not only those in the table) are in the sub$\mathrm{cm}^{-1}$ range. Time-dependent points were used. The KEO is written so that in each term derivatives (if any) are on the right and coefficients (which are evaluated at collocation points) are on the left. The PES is not a SOP of factors that depend on the coordinates in which we solve the Schrödinger equation.

TABLE I: Selected $\mathrm{CH}_{4}$ vibrational energies $\left(\mathrm{cm}^{-1}\right)$ compared with reference values given in the supplementary material of Ref. 81.

\begin{tabular}{lrrrr}
\hline \hline Assignment & Symmetry & $E_{\text {ref }}$ & $E_{\text {mctdh }}$ & $E_{\text {mctdh }}-E_{\text {ref }}$ \\
\hline & & 1310.47 & 1310.48 & 0.01 \\
$(00)(01)$ & $\mathrm{F}_{2}$ & 1310.47 & 1310.48 & 0.01 \\
& & 1310.47 & 1310.48 & 0.01 \\
& & 1533.47 & 1533.45 & -0.02 \\
$(00)(10)$ & $\mathrm{E}$ & 1533.47 & 1533.45 & -0.02 \\
& & 2586.99 & 2587.01 & 0.02 \\
$(00)(02)$ & $\mathrm{A}_{1}$ & 2613.83 & 2613.78 & -0.05 \\
& & 2613.83 & 2613.78 & -0.05 \\
$(00)(02)$ & $\mathrm{F}_{2}$ & 2613.83 & 2613.80 & -0.03 \\
& & 2624.44 & 2624.45 & 0.01 \\
& & 2624.44 & 2624.47 & 0.03 \\
$(00)(02)$ & $\mathrm{E}$ & 2830.20 & 2830.14 & -0.06 \\
& & & &
\end{tabular}




\begin{tabular}{|c|c|c|c|c|}
\hline & & 2830.20 & 2830.16 & -0.04 \\
\hline & & 2830.20 & 2830.16 & -0.04 \\
\hline & & 2845.96 & 2845.94 & -0.02 \\
\hline$(00)(11)$ & $\mathrm{F}_{1}$ & 2845.96 & 2845.95 & -0.01 \\
\hline & & 2845.96 & 2846.95 & -0.01 \\
\hline$(10)(00)$ & $\mathrm{A}_{1}$ & 2917.18 & 2917.09 & -0.09 \\
\hline & & 3019.43 & 3019.51 & 0.08 \\
\hline$(01)(00)$ & $\mathrm{F}_{2}$ & 3019.43 & 3019.51 & 0.08 \\
\hline & & 3019.43 & 3019.51 & 0.08 \\
\hline$\vdots$ & $\vdots$ & $\vdots$ & $\vdots$ & $\vdots$ \\
\hline & & 4224.19 & 4224.22 & 0.03 \\
\hline (10) (01) & $\mathrm{F}_{2}$ & 4224.19 & 4224.23 & 0.04 \\
\hline & & 4224.19 & 4224.23 & 0.04 \\
\hline & & 4319.00 & 4319.02 & 0.02 \\
\hline$(01)(01)$ & $\mathrm{F}_{2}$ & 4319.00 & 4319.02 & 0.02 \\
\hline & & 4319.00 & 4319.04 & 0.04 \\
\hline (01) $(01)$ & $\mathrm{F}$ & 4322.07 & 4322.08 & 0.01 \\
\hline (01) & $\mathbf{H}$ & 4322.07 & 4322.08 & 0.01 \\
\hline & & 4322.34 & 4322.41 & 0.07 \\
\hline$(01)(01)$ & $\mathrm{F}_{1}$ & 4322.34 & 4322.44 & 0.10 \\
\hline & & 4322.34 & 4322.45 & 0.11 \\
\hline$(01)(01)$ & $\mathrm{A}_{1}$ & 4322.71 & 4322.69 & -0.02 \\
\hline & & 4348.62 & 4348.55 & -0.07 \\
\hline$(00)(21)$ & $\mathrm{F}_{2}$ & 4348.62 & 4348.59 & -0.03 \\
\hline & & 4348.62 & 4348.59 & -0.03 \\
\hline & & 4363.59 & 4363.66 & 0.07 \\
\hline$(00)(21)$ & $\mathrm{F}_{1}$ & 4363.59 & 4363.68 & 0.09 \\
\hline & & 4363.59 & 4363.69 & 0.10 \\
\hline & & 4378.94 & 4378.88 & -0.06 \\
\hline$(00)(21)$ & $\mathrm{F}_{2}$ & 4378.94 & 4378.89 & -0.05 \\
\hline
\end{tabular}




\begin{tabular}{|c|c|c|c|c|}
\hline & & 4378.94 & 4378.89 & -0.05 \\
\hline \multirow{2}{*}{ (10) (10) } & \multirow{2}{*}{$\mathrm{E}$} & 4435.97 & 4435.93 & -0.04 \\
\hline & & 4435.97 & 4435.94 & -0.03 \\
\hline \multirow{3}{*}{ (01) (10) } & \multirow{3}{*}{$\mathrm{F}_{1}$} & 4537.52 & 4537.48 & -0.04 \\
\hline & & 4537.52 & 4537.48 & -0.04 \\
\hline & & 4537.52 & 4337.48 & -0.04 \\
\hline \multirow{3}{*}{ (01) (10) } & \multirow{3}{*}{$\mathrm{F}_{2}$} & 4543.81 & 4534.85 & 0.04 \\
\hline & & 4543.81 & 4534.89 & 0.08 \\
\hline & & 4543.81 & 4534.89 & 0.08 \\
\hline \multirow{2}{*}{ (00) (30) } & \multirow{2}{*}{$\mathrm{E}$} & 4592.13 & 4592.01 & -0.12 \\
\hline & & 4592.13 & 4592.06 & -0.07 \\
\hline (00) (30) & $\mathrm{A}_{2}$ & 4595.36 & 4595.39 & 0.03 \\
\hline (00) (30) & $\mathrm{A}_{1}$ & 4595.63 & 4595.59 & -0.04 \\
\hline
\end{tabular}

\section{CONCLUSION}

MCTDH is an increasingly popular method for solving the Schrödinger equation. Most MCTDH calculations have been done on PESs in SOP form, ${ }^{1}$ although it is also straightforward to use PESs represented with a truncated N-mode expansion each of whose terms is a SOP. ${ }^{47}$ The need to use one of these forms restricts the range of problems to which MCTDH can be applied. The first attempt to break free of the SOP form was the CDVR idea. ${ }^{48}$ Using the CDVR, one can apply MCTDH to any PES. C-MCTDH ${ }^{50}$ is an alternative that also enables one to use any PES. Both CDVR and C-MCTDH overcome an important weakness of the original MCTDH algorithm because they open the door to using general PESs.

In both the original CDVR and C-MCTDH, the basis functions are products of 1-D SPFs. If a direct product basis is used, the size of the basis set scales exponentially with the number of atoms in the system. An idea for reducing the size of the C-MCTDH basis was proposed in Ref. 51, where a pruned product basis is used with a Smolyak collocation grid. ${ }^{87,88}$ The pruned basis and Smolyak grid were orders of magnitudes smaller than their direct product 
counterparts. It has also been possible to combine CDVR with mode combination and multilayer ideas, both of which use multivariate SPFs, to avoid both exponential scaling of the basis size and obviate the need for a SOP PES. However, the combination is tricky because one needs to evaluate integrals in a product SPF basis with DVR ideas. It is straightforward to evaluate integrals in a basis of products of 1-D SPFs by transforming to a DVR. One assumes that all functions of coordinates are diagonal in the DVR and obtains quadrature-like equations. When the SPFs are multivariate, there is no DVR-like representation in which coordinate operators are diagonal. The best one can do is to nearly simultaneously diagonalise the coordinate operators. ${ }^{49,53}$ To obtain quadrature-like equations one must neglect off-diagonal elements. The best approach is one that introduces weights so that the most important off-diagonal matrix elements are the smallest. ${ }^{52}$

In this paper, we propose using $\mathrm{C}-\mathrm{MCTDH}$ in conjunction with mode combination ${ }^{17}$ so that the basis size does not scale exponentially with the number of atoms. Mode combination is a powerful idea because it incorporates all coupling between physical coordinates lumped into one logical coordinate into the SPF basis. Early in the history of MCTDH, mode combination made it possible to do calculations on molecules with dozens of coordinates. ${ }^{17}$ Because we use collocation, no integrals are required. We need only values of the PES at a set of collocation points. As collocation points, we use simultaneous diagonalisation (SD-)DVR points ${ }^{53}$ and Leja points, ${ }^{51,89,90}$ which are known to work well in conjunction with interpolation. ${ }^{61-64}$ When SD-DVR points are used with collocation there is no need to neglect off-diagonal matrix elements (and no need for the corresponding SD-DVR functions). Collocation requires only points, and when the basis is good enough the choice of the points is relatively unimportant. We have used a basis that is a direct product of multivariate SPFs, but it ought to be possible to use a nondirect product basis as well.

To test the new approach, we calculated accurate vibrational levels of $\mathrm{CH}_{3}, \mathrm{CH}_{4}$, and $\mathrm{CH}_{3} \mathrm{CN}$ by solving the time-independent Schrödinger equation using a collocation improved relaxation method. ${ }^{56}$ To derive the collocation improved relaxation equations, we used the idea of projecting the residual, that was employed in Ref. 50 .

Many impressive calculations have been done with mode combination, but with one exception they have all have been done with a SOP PESs. These calculations clearly demonstrate the effectiveness of the mode combination idea. The one exception is Ref. 49. In Ref. 49, an SD-DVR is used to compute photodissociation cross sections. It was later observed that 
neglecting off-diagonal elements in order to use a SD-DVR quadrature introduces significant errors in vibrational energy levels. ${ }^{52}$ In the ML-MCTDH context the errors could be reduced by introducing weights. In this paper, we show that the mode combination idea that is so effective with SOP PESs can be straightforwardly used with C-MCTDH.

Many new computational methods are designed to reduce the CPU and/or memory cost of calculations. The raison d'être of C-MCTDH, with or without mode combination, is different. Its most important advantage is that it makes it possible to do MCTDH calculations with a general PES. Nonetheless, in many cases C-MCTDH will also reduce the CPU cost of calculations because there is no need to evaluate MVPs for each term in the Hamiltonian separately. When using mode combination, it is difficult to make a concise statement about how the cost of the method scales with $D$. Combining many modes into the logical coordinates greatly reduces the cost of an MCTDH calculation, but increases the cost of solving the SPF equations for the combined modes. C-MCTDH, with or without mode combination, also has the advantage, at least when used to compute spectra, that time-independent points can be used. This works because if the basis is good enough the choice of the points is immaterial. The proviso is important. In some cases, we have observed that some energy levels are better converged when we use Leja points rather than SD-DVR points, which is a clear indication that in practice, at least if high accuracy is required, one does need to be careful about the selection of the points. Most MCTDH calculations are done to solve the time-dependent Schrödinger equation. It will be important to test our schemes for choosing points for time-dependent problems.

\section{DATA AVAILABILITY STATEMENT}

The data that support the findings of this study are available from the corresponding author upon reasonable request.

\section{Acknowledgements}

The financial support of the Natural Sciences and Engineering Research Council is gratefully acknowledged. We thank Compute Canada for providing computer time. 
1 M. H. Beck, A. Jäckle, G. A. Worth, and H.-D. Meyer, Physics Reports 324, 1 (2000).

2 H.-D. Meyer, F. Gatti, and G. A. Worth, Multidimensional Quantum Dynamics: MCTDH Theory and Applications (Weinheim: Wiley-VCH, 2009).

3 M. Nest and H.-D. Meyer, J. Chem. Phys. 119, 24 (2003).

${ }^{4}$ M. D. Coutinho-Neto, A. Viel, and U. Manthe, J. Chem. Phys. 121, 9207 (2004).

5 O. Vendrell, F. Gatti, D. Lauvergnat, and H.-D. Meyer, Angew. Chemie Int. Ed. 46, 6918 (2007)

6 T. Hammer, M. D. Coutinho-Neto, A. Viel, and U. Manthe, J. Chem. Phys. 131, 224109 (2009).

7 T. Hammer and U. Manthe, J. Chem. Phys. 134, 224305 (2011).

8 M. Schröder, F. Gatti, and H.-D. Meyer, J. Chem. Phys. 134, 234307 (2011).

9 T. Hammer and U. Manthe, J. Chem. Phys. 136, 054105 (2012).

10 A. Valdes, R. Prosmiti, and G. Delgado-Barrio, J. Chem. Phys. 137, 214308 (2012).

11 Q. Meng and H.-D. Meyer, J. Chem. Phys. 138, 014313 (2013).

12 M. Schröder and H.-D. Meyer, J. Chem. Phys. 141, 034116 (2014).

13 D. Peláez, K. Sadri, and H.-D. Meyer, Spectrochim. Acta: Part A 119, 42 (2014).

14 R. Wodraszka and U. Manthe, J. Phys. Chem. Lett. 6, 4229 (2015).

15 H.-D. Meyer, U. Manthe, and L. S. Cederbaum, Chem. Phys. Lett. 165, 73 (1990).

16 U. Manthe, H.-D. Meyer, and L. S. Cederbaum, J. Chem. Phys. 97, 3199 (1992).

17 G. A. Worth, H.-D. Meyer, and L. S. Cederbaum, J. Chem. Phys. 109, 3518 (1998).

18 H. Wang, J. Chem. Phys. 113, 9948 (2000).

19 A. Raab, G. A. Worth, H.-D. Meyer, and L. S. Cederbaum, J. Chem. Phys. 110, 936 (1999).

20 Z. Bacic and J. C. Light, Annu. Rev. of Phys. Chem. 40, 469 (1989).

21 S. Carter and N. C. Handy, Comput. Phys. Rep. 5, 117 (1986).

22 J. Tennyson, Comput. Phys. Rep. 4, 1 (1986).

23 T. Carrington, Jr., J. Chem. Phys. 146, 120902 (2017).

${ }^{24}$ H. Wang and M. Thoss, J. Chem. Phys. 119, 1289 (2003).

25 U. Manthe, J. Chem. Phys. 128, 164116 (2008).

26 O. Vendrell and H.-D. Meyer, J. Chem. Phys. 134, 044135 (2011).

27 R. Wodraszka and T. Carrington, Jr., J. Chem. Phys. 145, 044110 (2016). 
28 R. Wodraszka and T. Carrington, Jr., J. Chem. Phys. 146, 194105 (2017).

29 H. R. Larsson, B. Hartke, and D. J. Tannor, J. Chem. Phys. 145, 204108 (2016).

30 H. R. Larsson and D. J. Tannor, J. Chem. Phys. 147, 044103 (2017).

31 U. Manthe and H. Köppel, J. Chem. Phys. 93, 345 (1990).

32 M. J. Bramley and T. Carrington, Jr., J. Chem. Phys. 99, 8519 (1993).

33 H.-G. Yu and J. T. Muckerman, J. Mol. Spectrosc. 214, 11 (2002).

34 H. Han, A. Li, and H. Guo, J. Chem. Phys. 141, 244312 (2014).

35 S. A. Orszag, J. Comput. Phys. 37, 70 (1980).

36 J. P. Boyd, Chebyshev \& Fourier Spectral Methods (Dover Publications, 2001).

37 A. Jäckle and H.-D. Meyer, J. Chem. Phys. 104, 7974 (1996).

38 D. Peláez and H.-D. Meyer, J. Chem. Phys. 138, 014108 (2013).

39 M. Schröder and H.-D. Meyer, J. Chem. Phys. 147, 064105 (2017).

40 S. Manzhos and T. Carrington, Jr., J. Chem. Phys. 125, 194105 (2006).

41 S. Manzhos and T. Carrington, Jr., J. Chem. Phys. 127, 014103 (2007).

42 W. Koch and D. H. Zhang, J. Chem. Phys. 141, 021101 (2014).

43 E. Pradhan and A. Brown, J. Theor. Comput. Chem. 16, 1730001 (2017).

44 D. Hankins, J. W. Moskowitz, and F. H. Stillinger, J. Chem. Phys. 53, 4544 (1970).

45 S. Carter, S. J. Culik, and J. M. Bowman, J. Chem. Phys. 107, 10458 (1997).

46 X. Hu, T. Ho, and H. Rabitz, Comput. Phys. Commun. 113, 168 (1998).

47 O. Vendrell, F. Gatti, and H.-D. Meyer, J. Chem. Phys. 127, 184303 (2007).

48 U. Manthe, J. Chem. Phys. 105, 6989 (1996).

49 R. van Harrevelt and U. Manthe, J. Chem. Phys. 123, 064106 (2005).

50 R. Wodraszka and T. Carrington, Jr., J. Chem. Phys. 148, 044115 (2018).

51 R. Wodraszka and T. Carrington, Jr., J. Chem. Phys. 150, 154108 (2019).

52 U. Manthe, J. Chem. Phys. 130, 054109 (2009).

53 R. Dawes and T. Carrington, Jr., J. Chem. Phys. 121, 726 (2004).

54 D. Gottlieb and S. Orszag, Numerical Analysis of Spectral Methods (Society for Industrial and Applied Mathematics, 1977).

55 S. F. Boys, Proc. R. Soc. London, Ser. A 309, 195 (1969).

56 H.-D. Meyer, F. L. Quere, C. Leonard, and F. Gatti, Chem. Phys. 329, 179 (2006).

57 L. Doriol, F. Gatti, C. Iung, and H.-D. Meyer, J. Chem. Phys. 129, 224109 (2008). 
58 The Heidelberg MCTDH package, http://mctdh.uni-hd.de.

59 M. Powell, Approximation Theory and Methods (Cambridge University Press, 1981).

60 M. Briani, A. Sommariva, and M. Vianello, J. Comp. Appl. Math. 236, 2477 (2012).

61 M. Griebel and J. Oettershagen, J. Approximation Theory 207, 348 (2016).

62 P. Jantsch, C. G. Webster, and G. Zhang, IMA Journal of Numerical Analysis 00, 1 (2018).

63 A. Narayan and J. D. Jakeman, SIAM J. Sci. Comput. 36, 2952 (2014).

64 F. Leja, Ann. Polonici Math. 4, 8 (1957).

65 A. Edrei, ETNA 7, 124 (1998).

66 J. Balgama, D. Calvetti, and L. Reichel, ETNA 7, 124 (1998).

67 S. de Marchi, Appl. Math. and Comput. 152, 621 (2004).

68 A. Narayan and D. Xiu, SIAM J. Sci. Comput. 34, 1729 (2012).

69 A. Narayan, C. Gittelson, and D. Xiu, SIAM J. Sci. Comput. 36, 495 (2014).

70 G. Avila, J. Oettershagen, and T. Carrington, Jr., in Sparse Grids and Applications - Miami 2016 (Springer International Publishing, 2016), pp. 1-17.

71 U. Manthe, J. Chem. Phys. 128, 064108 (2008).

72 H.-D. Meyer and G. A. Worth, Theor. Chem. Acc. 109, 251 (2003).

73 G. H. Golub and C. F. Van Loan, Matrix Computations (The Johns Hopkins University Press, 1996).

74 E. R. Davidson, J. Comput. Phys. 17, 87 (1975).

75 R. B. Lehoucq, D. C. Sorensen, and C. Yang, ARPACK Users Guide: Solution of Large Eigenvalue Problems with Implicitly Restarted Arnoldi Methods (SIAM, Philadelphia, 1998).

76 M. H. Beck and H.-D. Meyer, Z. Phys. D 42, 113 (1997).

77 U. Manthe, Chem. Phys. 329, 168 (2006).

78 G. Avila and T. Carrington, Jr., J. Chem. Phys. 134, 054126 (2011).

79 D. Bégué, P. Carbonnière, and C. Pouchan, J. Phys. Chem. A 109, 4611 (2005).

80 D. M. Medvedev, L. B. Harding, and S. K. Gray, Molecular Physics 104, 73 (2006).

81 X.-G. Wang and T. Carrington, Jr., J. Chem. Phys. 141, 154106 (2014).

82 F. T. Smith, Phys. Rev. Lett. 45, 1157 (1980).

83 G. Schiffel and U. Manthe, J. Chem. Phys. 132, 084103 (2010).

84 R. Wodraszka, J. Palma, and U. Manthe, J. Phys. Chem. A 116, 11249 (2012).

85 P. S. Thomas and T. Carrington, Jr., J. Phys. Chem. A 119, 13074 (2015). 
86 A. Leclerc and T. Carrington, Jr., J. Chem. Phys. 140, 174111 (2014).

87 G. Avila and T. Carrington, J. Chem. Phys. 139, 134114 (2013).

88 G. Avila and T. Carrington, J. Chem. Phys. 143, 214108 (2015).

89 G. Avila and T. Carrington, J. Chem. Phys. 147, 144102 (2017).

90 E. Żak and T. Carrington, Jr., J. Chem. Phys. 150, 204108 (2019). 